\title{
Desert Dust Properties, Modelling, and Monitoring
}

\author{
Dimitris G. Kaskaoutis, ${ }^{1}$ Ralph A. Kahn, ${ }^{2}$ Pawan Gupta, ${ }^{2,3}$ \\ Achuthan Jayaraman, ${ }^{4}$ and Aristides Bartzokas ${ }^{5}$
}

\author{
${ }^{1}$ Sharda University, Greater Noida 201306, India \\ ${ }^{2}$ Goddard Space Flight Center, NASA, Greenbelt, MD 20771, USA \\ ${ }^{3}$ Universities Space Research Association, Greenbelt, MD 20770, USA \\ ${ }^{4}$ National Atmospheric Research Laboratory, Gadanki 517112, India \\ ${ }^{5}$ Laboratory of Meteorology, University of Ioannina, 45110 Ioannina, Greece
}

Correspondence should be addressed to Dimitris G. Kaskaoutis, dkask@meteo.noa.gr

Received 10 October 2012; Accepted 10 October 2012

Copyright (c) 2012 Dimitris G. Kaskaoutis et al. This is an open access article distributed under the Creative Commons Attribution License, which permits unrestricted use, distribution, and reproduction in any medium, provided the original work is properly cited.

Dust storms are considered natural hazards that affect the earth's atmosphere and ecosystems over both short- (few hours to a few days) and long-time intervals. Due to the significant impact of dust aerosols on climate, air quality, and entire biota, different instrumentation and techniques have been used to focus on the investigation of such events. In order to improve the scientific understanding of dust aerosols, their source regions, and their effect on global climate, a worldwide effort has been undertaken in the last three decades to produce a global dust-aerosol climatology by combining satellite observations and measurements from ground-based monitoring networks. The studies included in this special issue mainly concern ground-based and satellite observations as well as model simulations of dust plumes over climatically sensitive areas influenced by dust storms, such as the tropical Atlantic, Mediterranean, Alps, northwestern India, and east Asia.

N. E. Touré et al. analyzed the intercontinental transport and climatic impact of the Saharan and Sahelian dust traversing the tropical Atlantic from the African coast towards the Caribbean and south America by means of the regional climate model (RegCM 4.0). The dust simulation was coherent with observations from MISR and AERONET, highlighting the importance of model simulations for estimating the dust radiative impact.

From two years of CALIPSO liar observations, D. Liu et al. studied the vertical andhorizontal spatial distribution of Saharan dust plumes traveling the Atlantic Ocean,and their seasonal variation. This study provides new insights into
Saharan dusttransport, which can be especially useful for future simulations of dust productionand transport.

G. Pavese et al. examined the effects of Saharan dust optical and physical properties (aerosol optical depth, Angstrom coefficients, and volume size distribution) in the western Mediterranean region using observations at five locations, three in south Italy, and two in southern Spain, conducted during eight campaigns of various duration.

P. T. Nastos analyzed the meteorological patterns associated with intense Saharan dust outbreaks over Greece during the winter season. He provided detailed analysis of surface pressure and geopotential height synoptic weather maps from NCEP/NCAR reanalysis during the dusty days in order to investigate the influence of the meteorology on dust emission, uplift, transport, and deposition. These results are supported by DREAM model simulations.

In a related study, D. G. Kaskaoutis et al. analyzed the same dust cases over eastern Mediterranean and Greece in the winter season (4-6 February, 2009) with the synergistic use of multiple satellite sensors (MODIS, OMI, and CALIPSO), focusing on the detection of the dust source regions, vertical distribution of the dust plume, and characteristics during the whole dusty cycle.

F. Calastrini et al. investigated a dust event in the centralwestern Mediterranean by the synergistic use of meteorological and aerosol optical depth observations. Mainly, they highlight the importance of models to better distinguish dust outbreaks that transport aerosol above the boundary layer and do not impact ground level from those causing regional 
deposition. They suggest an ad hoc model along with chemical and physical analysis to measure the concentration of soil-related elements and to quantify the contribution of dust particles to $\mathrm{PM}_{10}$.

F. Thevenon et al. examined the Saharan source regions and transport pathways of dust plumes that affect the highaltitude Alpine stations as well as the deposition of dust on Alpine glaciers using radiogenic isotope signatures and backward trajectory analysis.

The radiative forcing at the surface, top-of-atmosphere, and within the atmosphere caused by dust events was investigated over Patiala, northwestern India by D. Sharma et al. For this analysis, they used ground-based sun photometer and satellite-retrieved aerosol optical properties along with OPAC and SBDART models for the simulation of aerosol spectral optical properties and estimation of radiative forcing, respectively. They found that the dust events cause pronounced cooling at the surface and heating of the lower and middle troposphere, which may significantly influence regional climate, melting Himalayan glaciers and redistributing monsoonal rainfall.

The horizontal and vertical dynamical structure of Saharan dust was analyzed with a combination of satellite and ground-based observations as well as model (WRFChem) simulations (C. Cavazos-Guerra and M. C. Todd). The comparison between observations and WRF-Chem simulations highlighted the model efficiency at simulating the spatial and 3D structure of dust transport over the Sahara and Sahel, suggesting that accurate forecasting of dust events associated with synoptic scale events is possible when dust emission parameterization is suitably calibrated.

The work of X. Xi and I. N. Sokolik examines the impact of Asian dust on vegetation over dryland ecosystems by altering the photosynthetically active radiation (PAR) as well as shortwave and longwave radiation components. They also analyzed the radiative forcing efficiency of dust in the surface radiative balance.

I. Takemi examined the representations of shallow and deep convection under two distinct stability conditions over the Gobi desert using numerical outputs from largeeddy simulations. The results suggest that the adequate representations of shallow and deep convection are critically important in simulating the transport of dust aerosols under convective conditions.

D. Ackerley et al. have attempted to identify the differences arising in the representation of dust in a General Circulation Model (GCM) using two separate uplift schemes (CLIM and DEAD) over the globe. The study also allows a comparison between the dust uplift schemes without the added complexity of having several different transport and deposition models and helps explain how each uplift model responds to the simulated GCM. The dust simulation is particularly sensitive to (a) the dust-uplift parameterization scheme, (b) the representation of surface soil characteristics, and (c) the surface wind speed.

Finally, Mona et al. reviewed the use of lidar (light detection and ranging) techniques for the detection of optical properties as well as for the vertical distribution of dust. They highlight the benefits of lidar for the spatiotemporal monitoring of dust aerosol properties at various altitudes in the atmosphere, specifically, the intrusion into the planetary boundary layer and the mixing processes within the atmosphere. This review summarizes most of the information about desert dust collected to date using lidar techniques, and discusses the synergy of lidar with other remote sensing and in situ techniques.

Dimitris G. Kaskaoutis Ralph A. Kahn Pawan Gupta Achuthan Jayaraman Aristides Bartzokas 

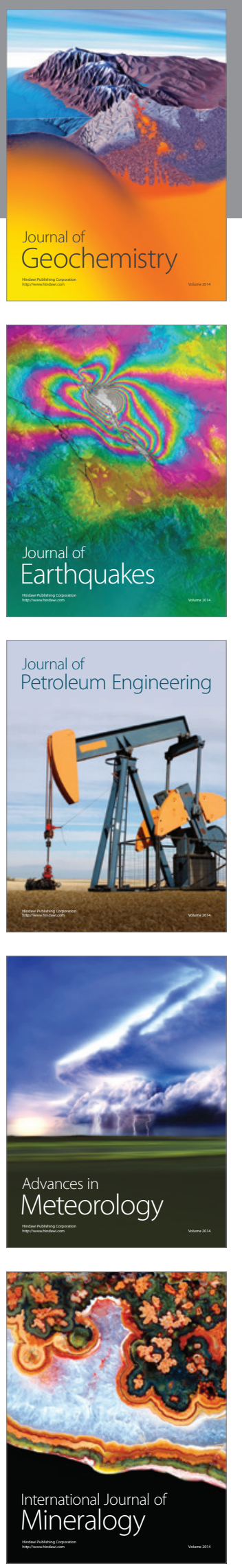
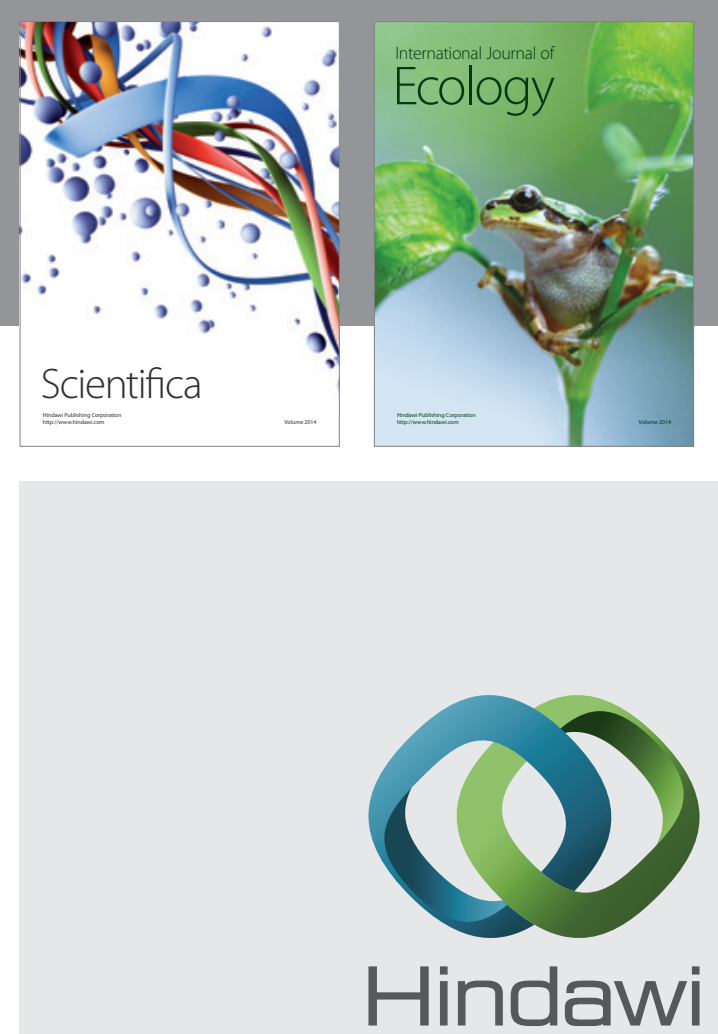

Submit your manuscripts at http://www.hindawi.com
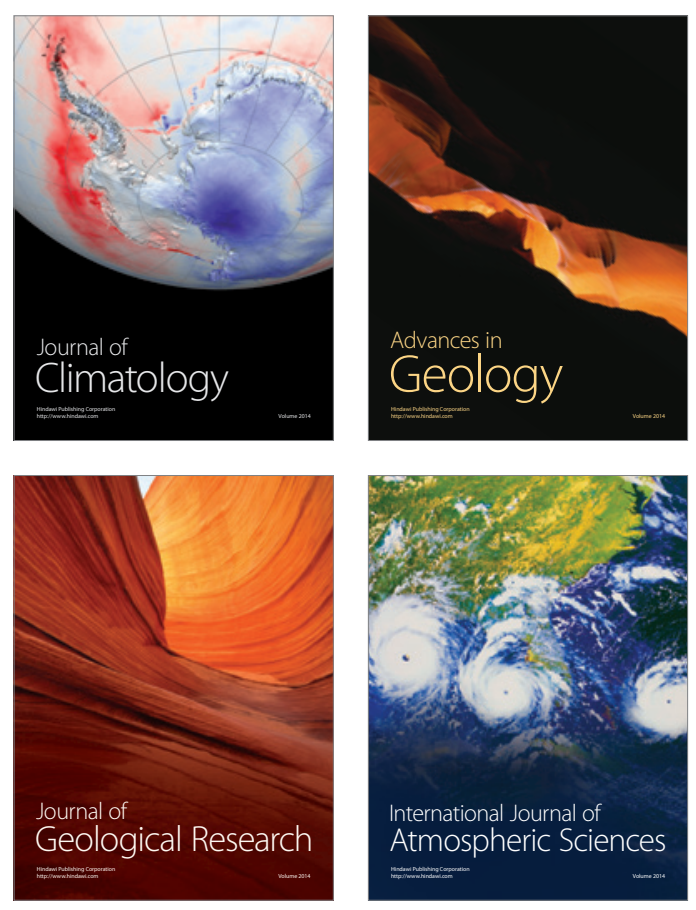
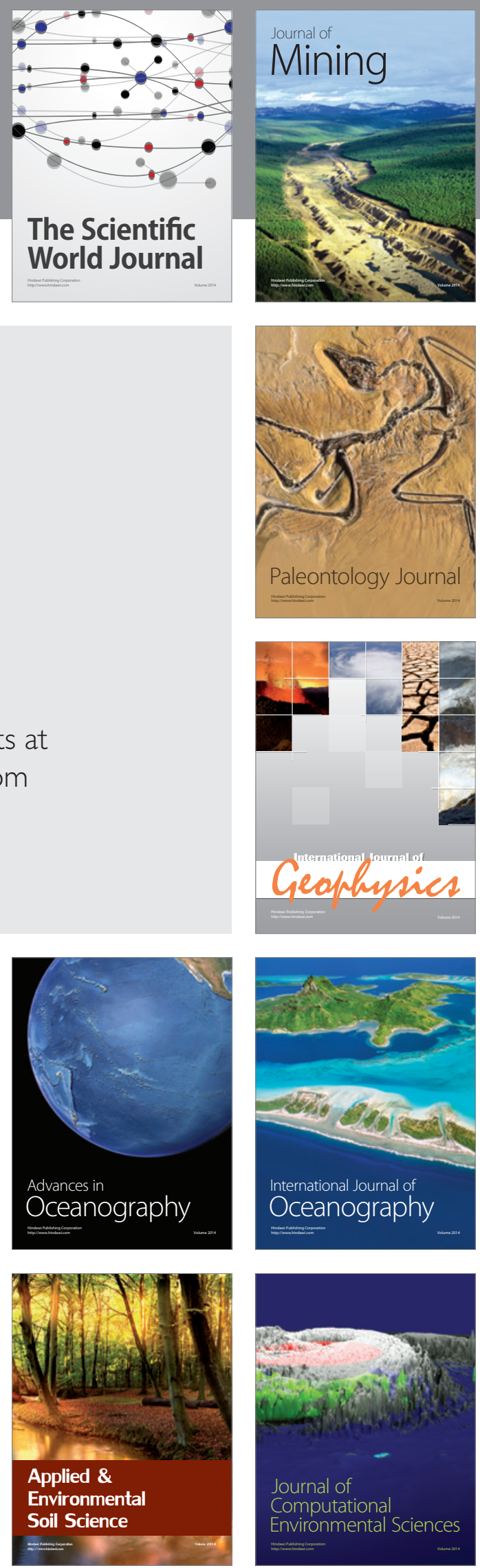